\title{
SUPPLEMANTARY INFORMATION \\ Orthogonal degron system for controlled protein degradation in cyanobacteria
}

Jonathan K. Sakkos ${ }^{1}$, Sergio Hernandez-Ortiz², Katherine W. Osteryoung ${ }^{3}$, Daniel C. Ducat'1,4, *

${ }^{1}$ MSU-DOE Plant Research Laboratory, Michigan State University, East Lansing, MI 48824, USA

2 Department of Microbiology \& Molecular Genetics, Michigan State University, East Lansing, MI 48824, USA

${ }^{3}$ Department of Plant Biology, Michigan State University, East Lansing, MI 48824, USA

${ }^{4}$ Department of Biochemistry \& Molecular Biology, Michigan State University, East Lansing, MI 48824, USA

*Corresponding author: email - ducatdan@msu.edu, phone - (517) 432-5118

\section{Contents}

Figure S1: Flow cytometry of $m f$-lon induction, Figure S2: Western blot showing $m f$-lon accumulation, Figure S3: mNG-PDT downregulation timecourse, Figure S4: Site-directed mutagenesis of the PDT, Figure S5: Effect of theophylline on growth rate,

Figure S6: Cartoon of expected phenotypes

Figure S7: Alternative FtsZ mutant hyperelongation

Figure S8: Control of cell elongation with truncated PDT

Figure S9: Genetic constructs for carboxysome manipulation

Figure S10: CcmL downregulation

Figure S11: Redistribution of carboxysome spacing after CcmO downregulation

Figure S12: Representative cell segmentations

Figure S13: Cytosolic RbcS depletion after $\mathrm{CcmO}$ downregulation

Table S1: Gene ontology enrichment analysis of $m f$-lon off-target proteins

Methods S1: Proteomics extended methods 


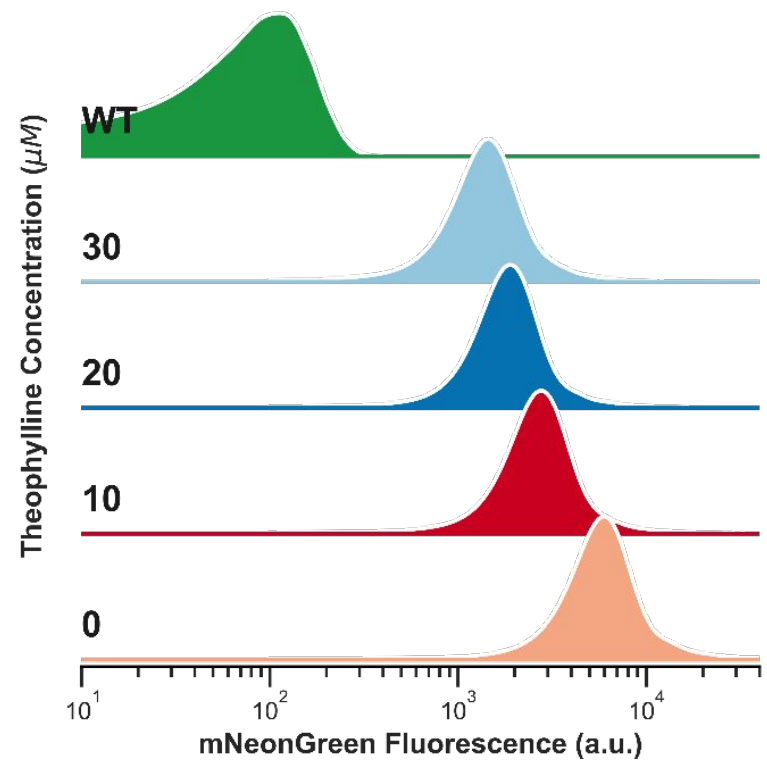

Figure S1: Population-level mNeonGreen-PDT reporter fluorescence following $m f$-lon protease induction at different inducer concentrations. Data shown was collected 24 hours following inducer addition (theophylline concentrations: $0,10,20$, or $30 \mu \mathrm{M}$ ) to strain DD309 (mNG-PDT/mf-Ion) and fluorescence measurements were obtained by flow cytometry. All curves are kernel density estimation fits to representative data from at least $n>9,000$ cells. Wild-type $S$. elongatus was also measured as a control for basal autofluorescence. Strains: wt and mNG-PDT/mf-Ion (DD309).

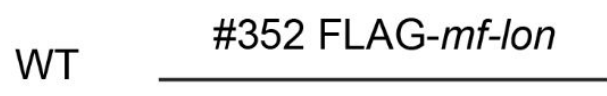

Theophylline $(\mu \mathrm{M}) \quad-\quad \begin{array}{llll}0 & 10 & 20 & 30\end{array}$

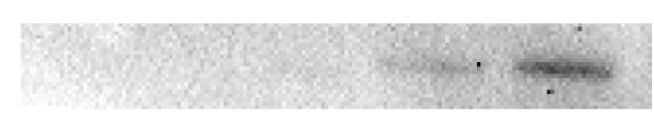

Figure S2: The abundance of $m f$-lon can be tuned in a dose-dependent manner based on theophylline induction. Immunoblot of the N-terminal FLAG epitope fusion to mf-lon after 24 hours of induction with the indicated concentration of theophylline. Strains: DD352, wt. 


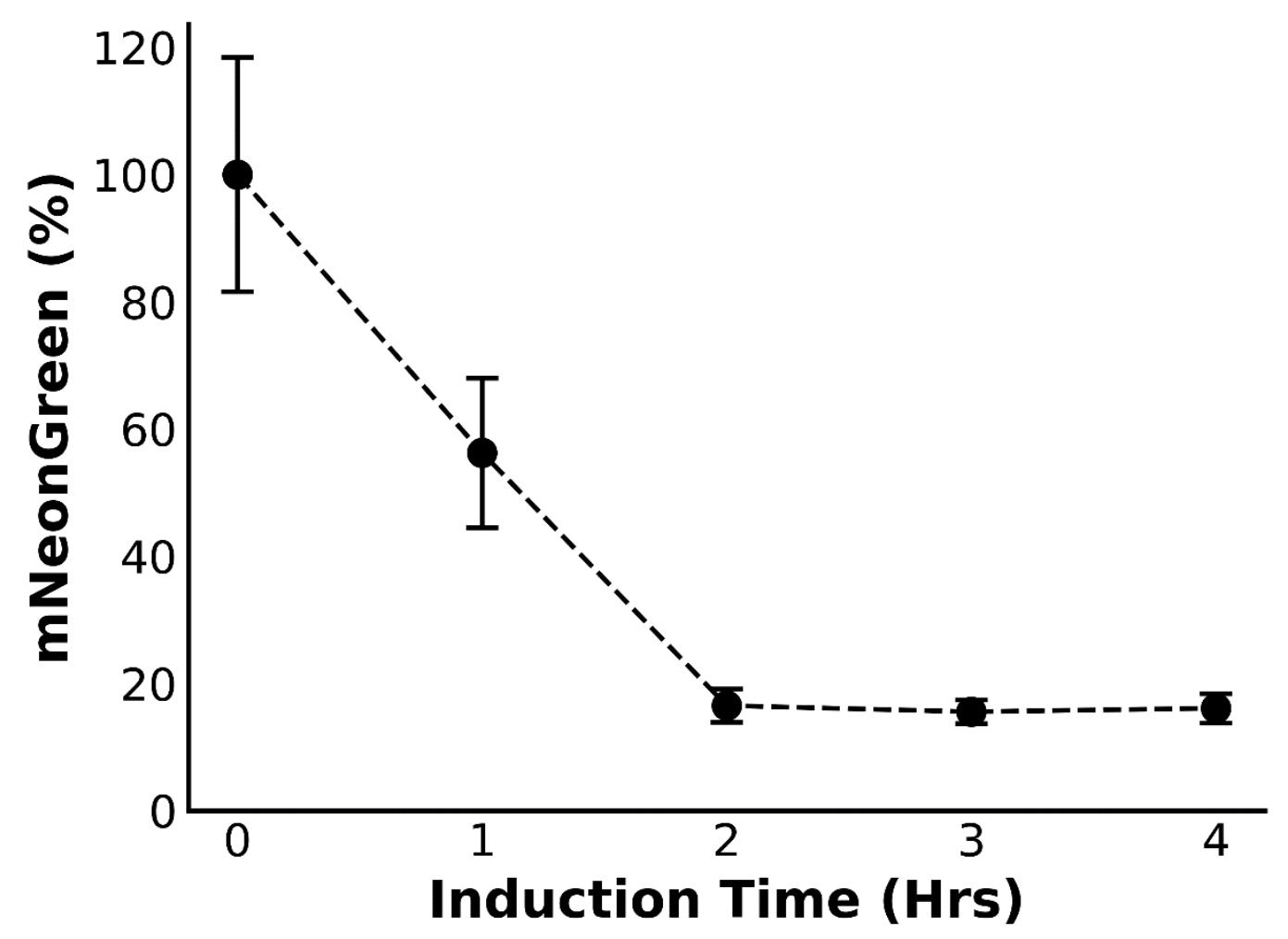

Figure S3: Rapid downregulation of PDT-tagged $\mathrm{mNeonGreen} \mathrm{reporter} \mathrm{under} \mathrm{high} \mathrm{mf-lon} \mathrm{induction.} 1 \mathrm{mM}$ theophylline was added to strain mNG-PDT/mf-Ion (DD309) at time zero and reporter intensity was measured by fluorescence microscopy in the following hours. The intensity was normalized by the signal prior to induction $(\mathrm{t}=0)$. Error bars represent standard deviation from $\geq 3$ replicates. Strains: $\mathrm{mNG}-\mathrm{PDT} / \mathrm{mf}$ Ion (DD309), 


\section{PDT 1- 1 AANKNEENTNEVPTFMLNAGQANRRRV-27}

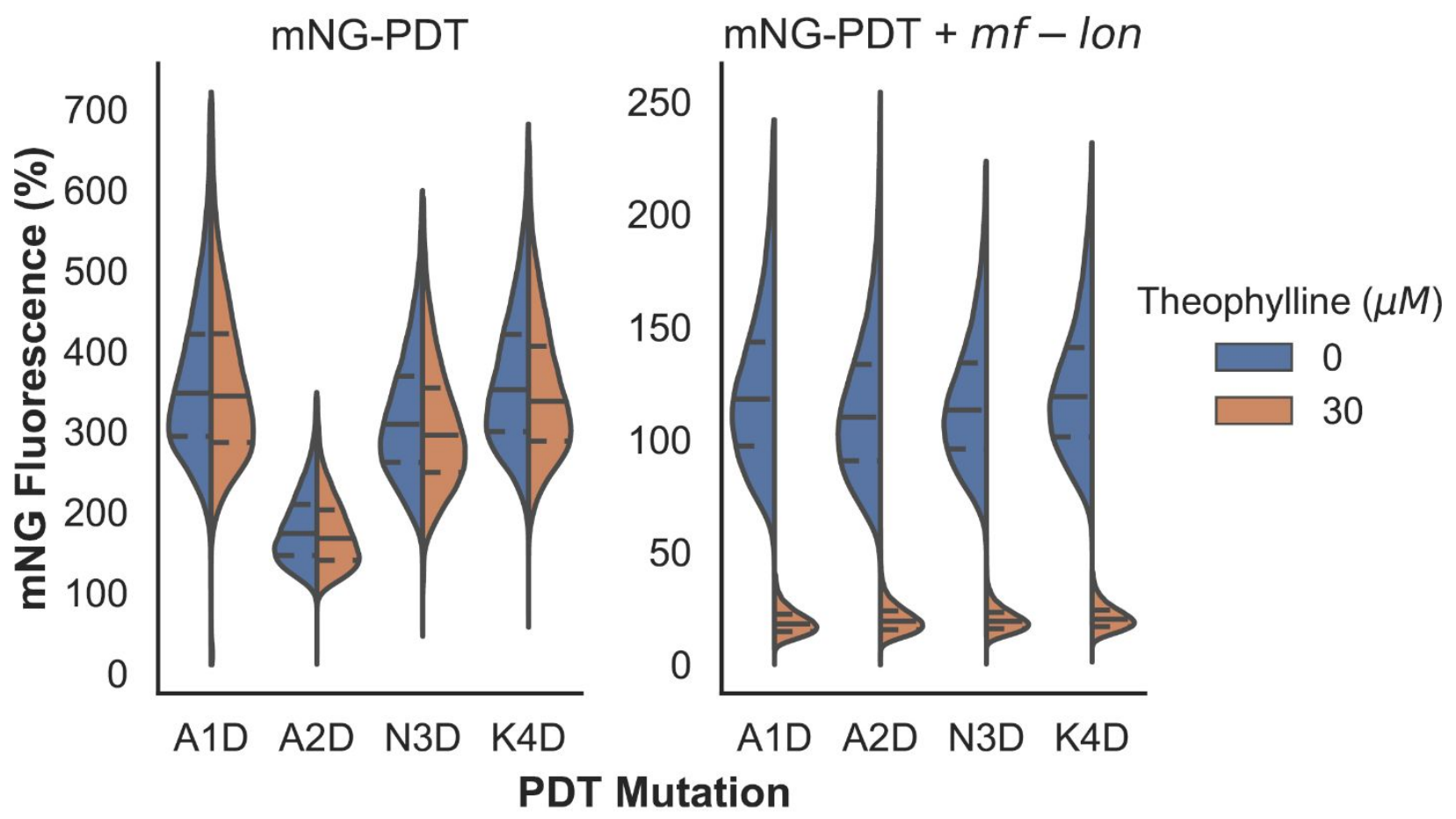

Figure S4: Relative fluorescence after 24 hours of $m$ NeonGreen $(m N G)$ reporters with modified PDT sequences, with and without heterologous expression of $m f$-lon protease. All values are normalized relative to the fluorescence intensity of the unmodified PDT sequence (displayed in top panel, and encoded in strain DD309; characterized in greater detail in main figure panels) in the absence of $m f$-lon induction. Site directed mutagenesis of the 4 amino acid residues at the $\mathrm{N}$-terminus of the PDT (AANK) that are similar to the ssrA degradation tag of $S$. elongatus resulted in a slightly higher basal expression of the reporter protein. However, all mNG-PDT variant reporters exhibited a lower basal fluorescence when examined in a strain containing the riboswitch-controlled $m f$-lon gene in comparison to the same construct in a WT background (mNG-PDT). Strains: mNG-PDT(A1D, DD315), mNG-PDT(A2D, DD316), mNG-PDT(N3D, DD317), mNGPDT(K4D, DD318), mNG-PDT(A1D)/mf-lon (DD322), mNG-PDT(A2D)/mf-lon (DD319), mNG$\mathrm{PDT}(\mathrm{N} 3 \mathrm{D}) / \mathrm{mf}$-lon (DD320), and mNG-PDT(K4D)/mf-lon (DD324). 


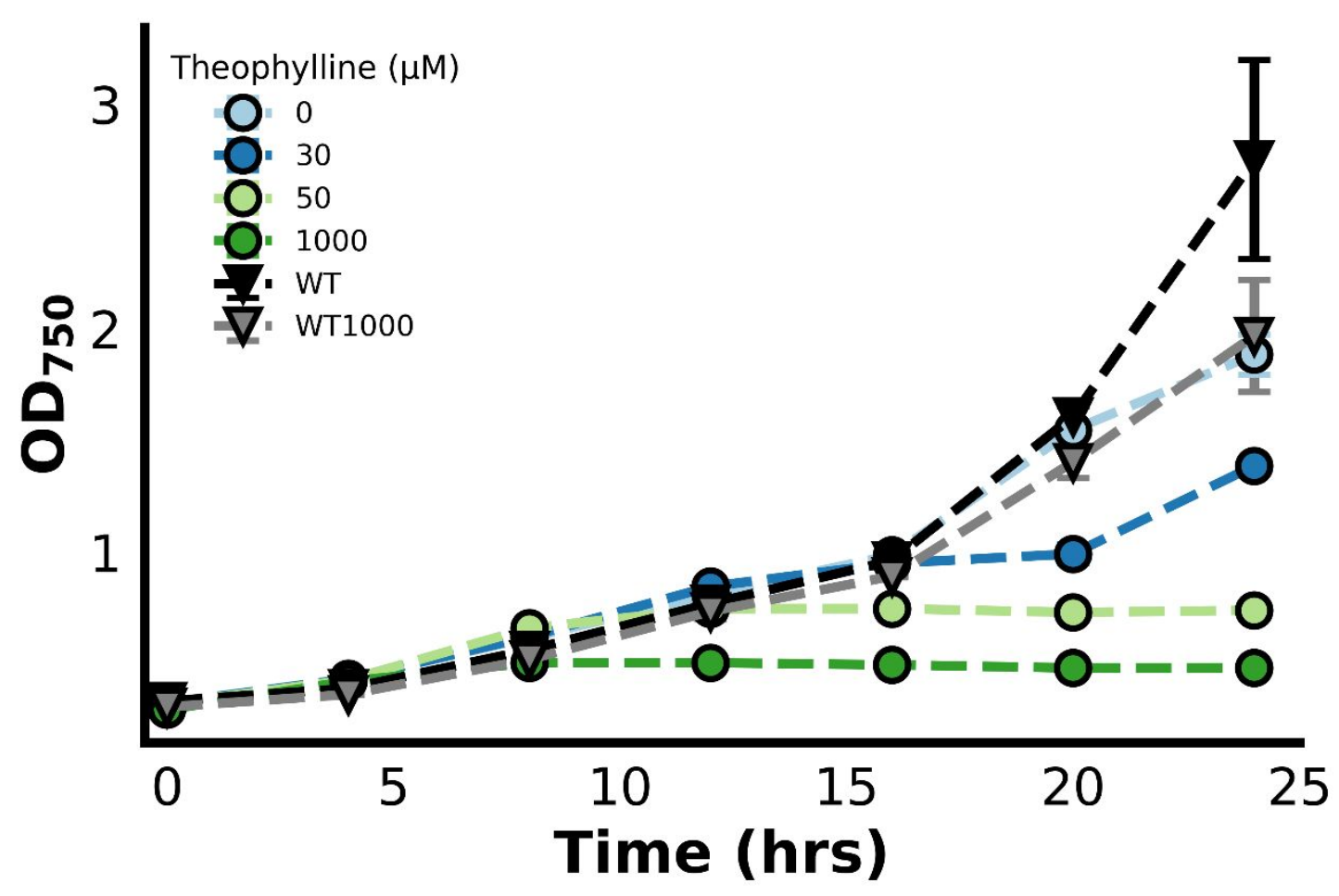

Figure S5: Growth curves of $S$. elongatus cultures when induced with increasing concentrations of theophylline. Wild-type controls (triangles) exhibited a slight growth defect with increased theophylline addition, as we have previously reported ${ }^{1}$. In contrast, the $\mathrm{mf}$-lon strain (DD206; circles) was hypersensitive to theophylline addition, exhibiting growth arrest at concentrations $>50 \mu \mathrm{M}$. Minor impairment of growth at higher cell densities $\left(\mathrm{OD}_{750}>1.5\right)$ was also observed in the mf-lon strain without induction. Error bars represent the standard deviation from 3 biological replicates. Strains: wt and $m f-l o n$ (DD206). 
A

WT cell length

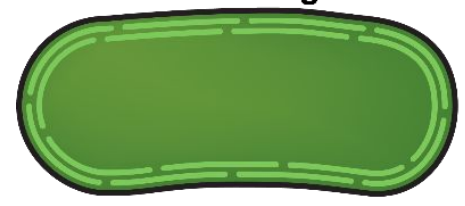

B

WT carboxysomes

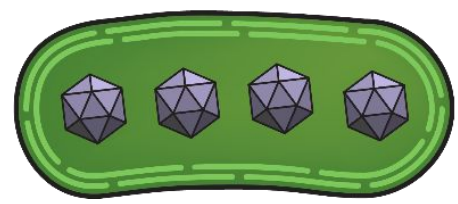

$\Delta c c m O$

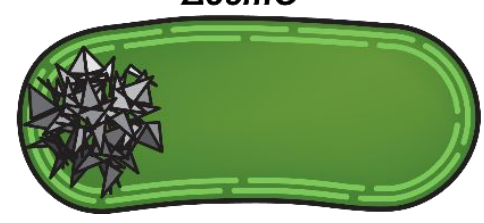

$\Delta f t s Z$

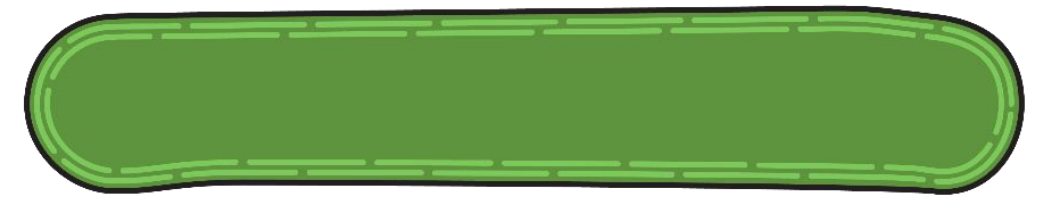

$\Delta c c m L$

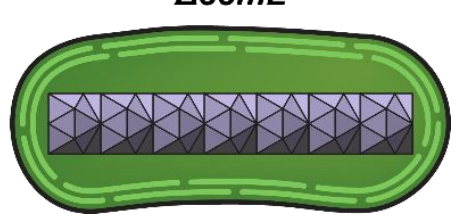

Figure S6: Cartoon schematic of expected cellular phenotypes resulting from gene deletion or protein downregulation. A) Disruption of FtsZ inhibits cell division while cell wall synthesis continues, leading to extreme cell elongation (e.g., see ${ }^{1}$ ), which is characteristic of strain FtsZ-PDT*/ mf-lon (DD347), shown in Figure 4, as well as the additional mutants shown in Figure S7 B) Deletion of either ccmO or ccmL impairs the formation of carboxysomes; $\triangle c c m O$ mutants display a round polar body of aggregated RuBisCO and remaining shell proteins while $\Delta c c m L$ mutants are unable to cap the carboxysome vertex and form bar-like structures ${ }^{2}$. CcmO-PDT/mf-lon/RbcS-mNG (DD321) is shown in Figures 5 and 6, while CcmL-PDT/mfIon/RbcS-mNG (DD348) is shown in Figure S10. 

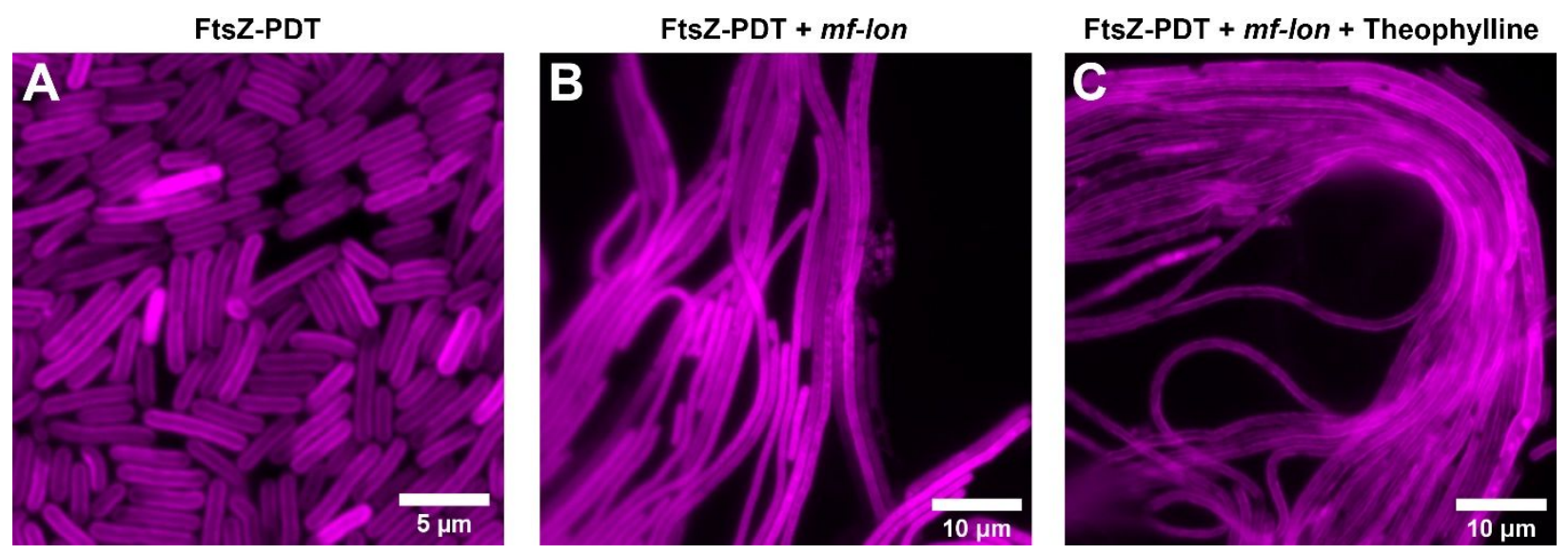

Figure S7: Leaky expression of mf-lon destabilizes FtsZ-PDT and presents a hyper elongated cell phenotype. A) Integration of FtsZ-PDT at the native genomic locus (strain DD346) leads to cells with near wild type length. B) However, integration of the same construct within a $m f$-lon background (i.e., strain DD312) generates strains with hyper elongated cells, typical of a FtsZ-disrupted phenotype. C) Addition of theophylline $(30 \mu \mathrm{M})$ to this strain (DD312) can exacerbate this phenotype (24 hours post induction). Scale bars as indicated. Strains: FtsZ-PDT (DD346), FtsZ-PDT/mf-Ion (DD312). 


\section{PDT* $^{*}$ \\ \begin{tabular}{r|r|}
1 & 14 \\
\hline AANKNEENTNEVP & TFMLNAGQANRRRV \\
\hline
\end{tabular}}
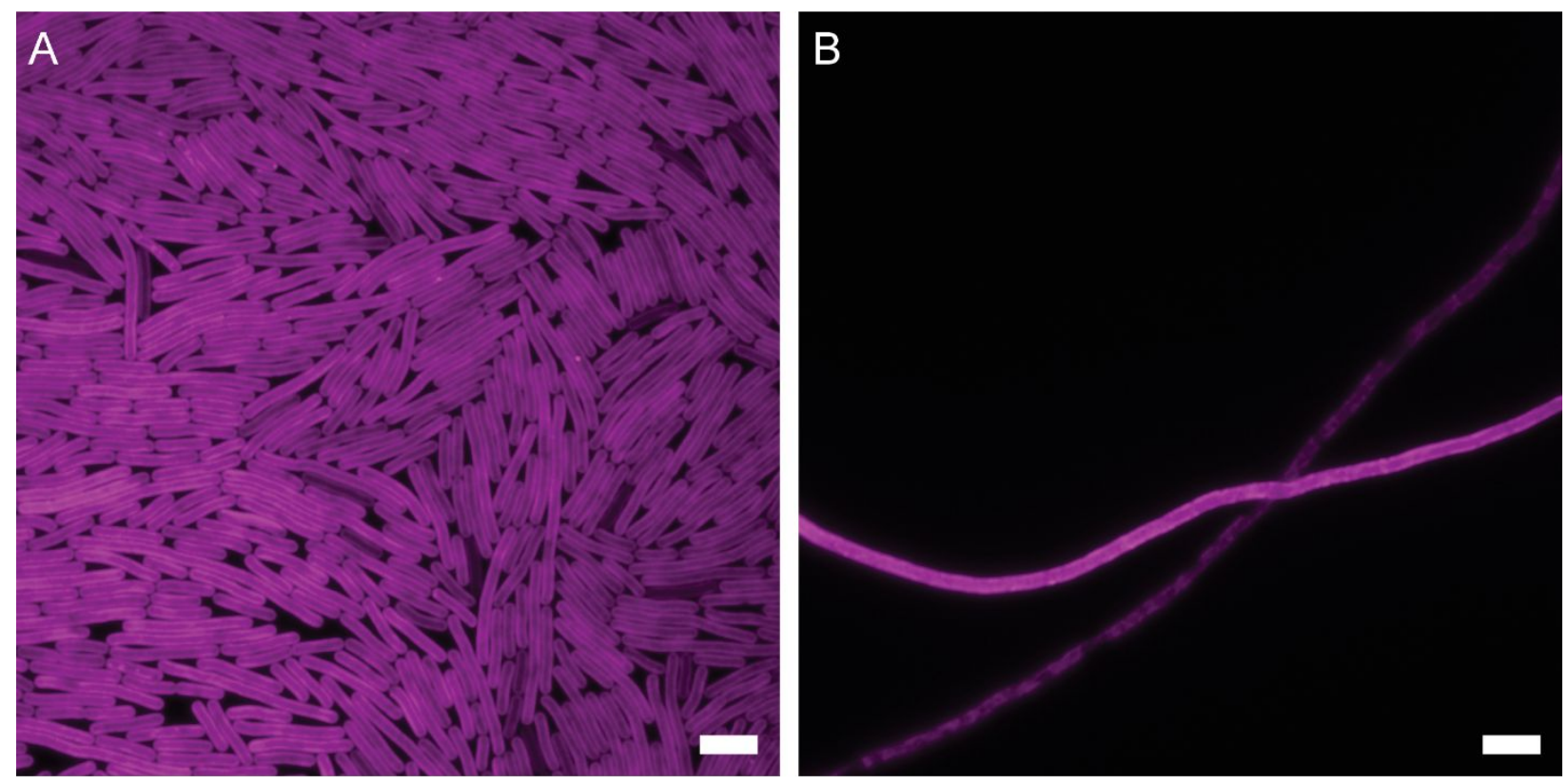

Figure S8: Truncated PDT (PDT*, sequence highlighted in red in top panel) enables control over Fts $Z$ cell elongation phenotype. A) Representative image of FtsZ-PDT*/mf-Ion (Strain DD347) in the absence of theophylline. B) The same strain after induction with $30 \mu \mathrm{M}$ theophylline for 48 hours. Scale bars are $5 \mu \mathrm{m}$. Strains: FtsZ-PDT*/mf-Ion (DD347). 

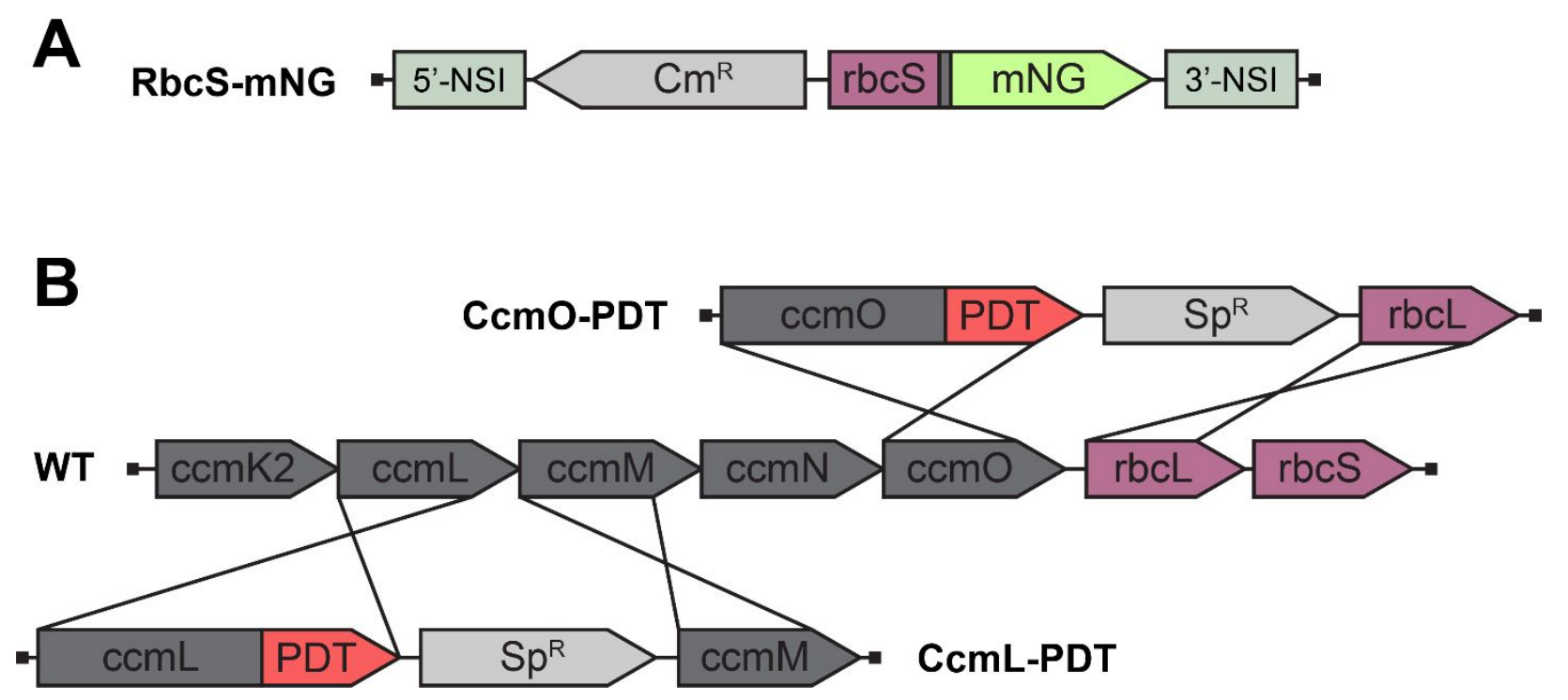

Figure S9: Genetic constructs used to regulate carboxysome shell protein stability via $m f$-lon targeting. A) For all carboxysome-related strains, $\mathrm{mNeonGreen} \mathrm{(} \mathrm{mNG}$ ) was fused with a heterologously-expressed copy of $r b c S$ (RbcS-mNG) inserted into Neutral Site 1 (NSI). B) Native copies of the targeted carboxysome shell proteins ( $\mathrm{cmO}$ and $\mathrm{ccmL}$ ) were tagged with the indicated PDT fusions in the native genomic context. $\mathrm{Cm}^{\mathrm{R}}$, chloramphenicol resistance; $\mathrm{Sp}^{\mathrm{R}}$, spectinomycin resistance. 


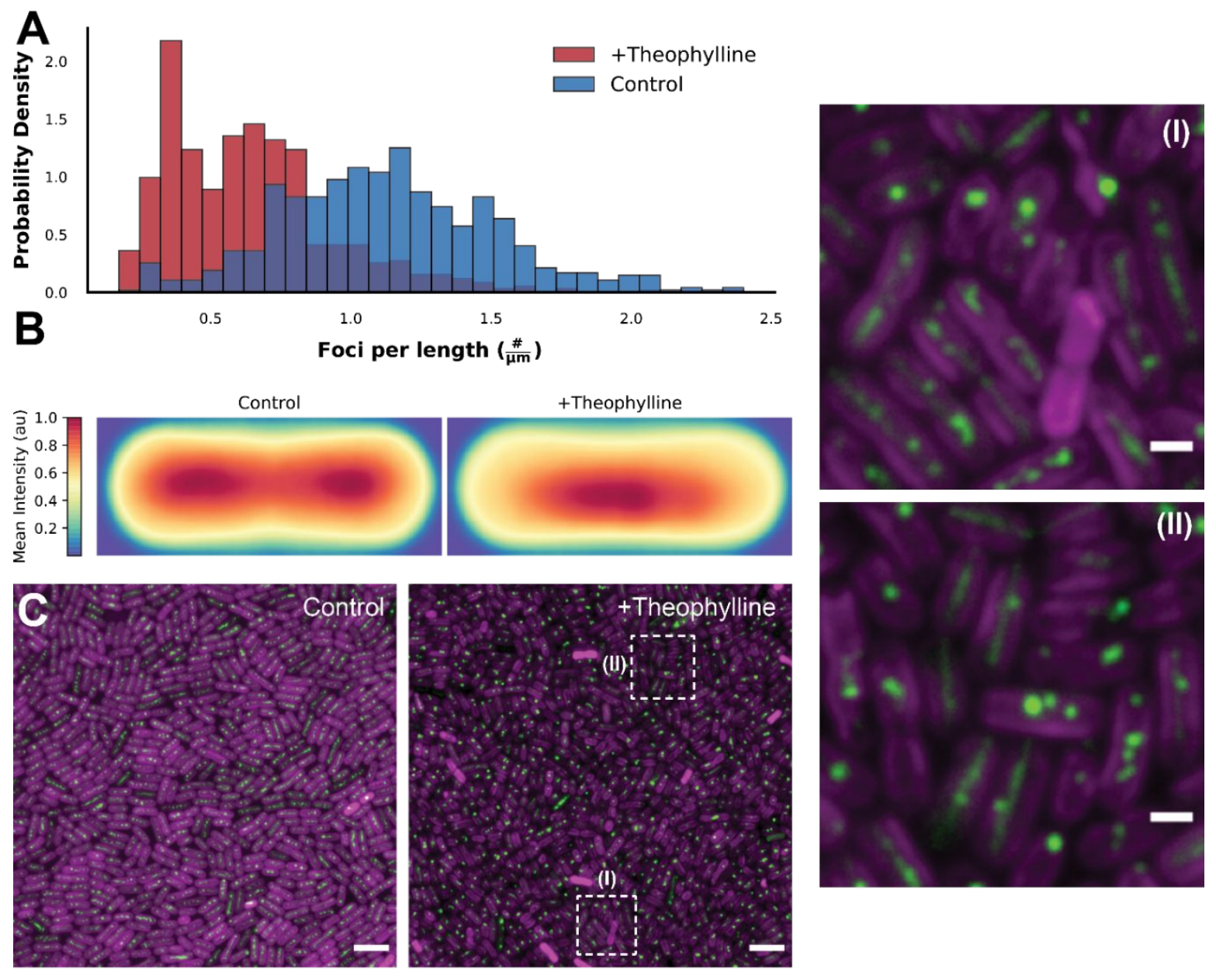

Figure S10: Carboxysome structure is reorganized following $m f$-lon induction in strains with PDT-tagged $\mathrm{CcmL}$. A) CcmL-PDT/mf-lon/RbcS-mNG strain (DD348) induced with $30 \mu \mathrm{M}$ theophylline exhibited fewer carboxysomes per cell as quantified 72 hours-post induction. B) Average fluorescence intensity distribution of carboxysome reporter (RbcS-mNG) in CcmL-PDT cells with (+theophylline; $30 \mu \mathrm{M}$ ) and without (control) induction of mf-lon (72 hours post induction). Carboxysome morphology and positioning after downregulation of CcmL-PDT shows irregular carboxysome shape, including frequent "bar carboxysomes" (rods), but carboxysomes are not concentrated towards poles as in $\mathrm{CcmO}$ downregulated cells. C) Representative fluorescence microscopy images of a CcmL-PDT/mf-lon/RbcS-mNG (DD348) 72 hours following mf-Ion induction (30 $\mathrm{MM}$; “+Theophylline") or in uninduced control. Insets show examples of the rod-like carboxysome phenotype. These phenotypes are consistent with published $\Delta c c m L$ strains ${ }^{2}$ Scale bars $5 \mu \mathrm{m}$ and $1 \mu \mathrm{m}$ (inset). A/B) $n=6,856$ (control), $n=808$ (+theophylline). Strains: CcmL-PDT/mfIon/RbcS-mNG (DD348). 


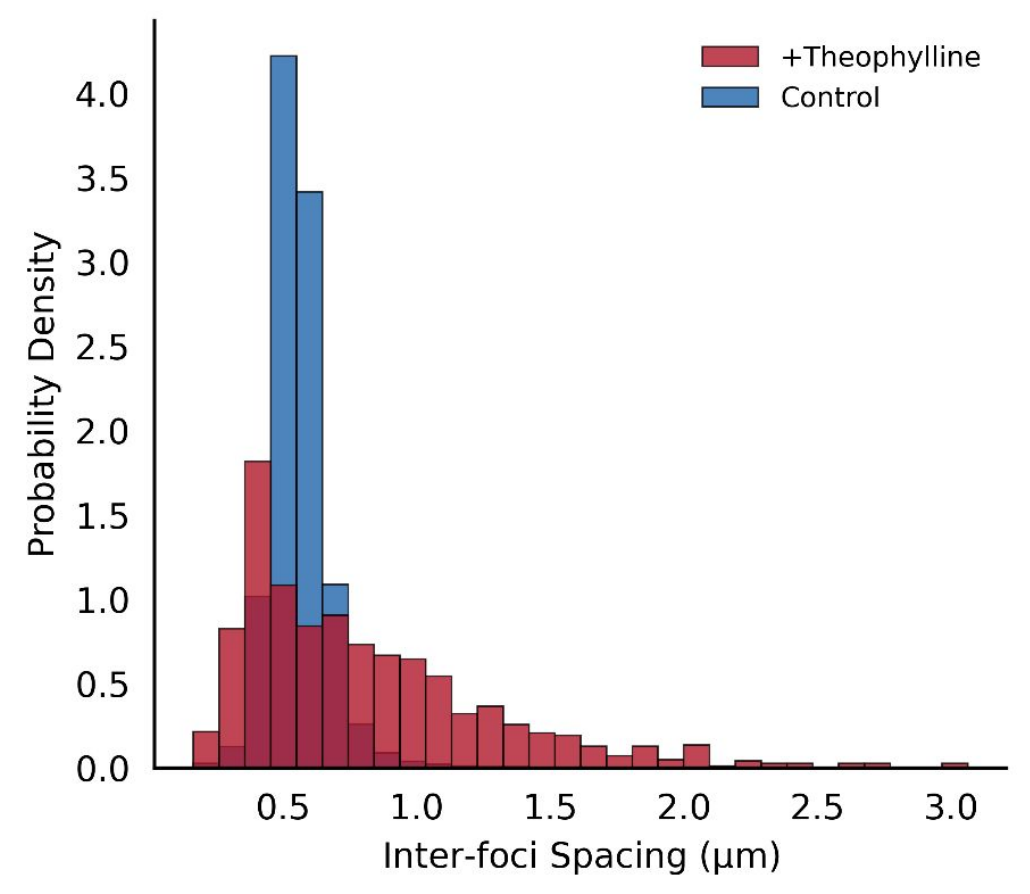

Figure S11: Carboxysome phenotype and polar localization is exacerbated 72 hours following mf-lon induction in CcmO-PDT strains. Strain DD321 (CcmO-PDT/mf-lon/RbcS-mNG) was induced with $30 \mu \mathrm{M}$ theophylline to target $\mathrm{CcmO}$ for degradation and carboxysome spacing was measured in $\mathrm{n}=6,838$ (control), $n=2,637$ (+theophylline) cells 72 hours after induction. For these measurements, it should be noted that only cells with more than one carboxysome were used in order to determine an inter-foci carboxysome spacing. A dominant phenotype was a single polar body of RbcS-mNG at this time point, but these cells were not included for this analysis. See Figure $6 \mathrm{D}$ for quantification of earlier time points and see Figure S11 for representative cell images. Stains: CcmO-PDT/mf-Ion/RbcS-mNG (DD321) 

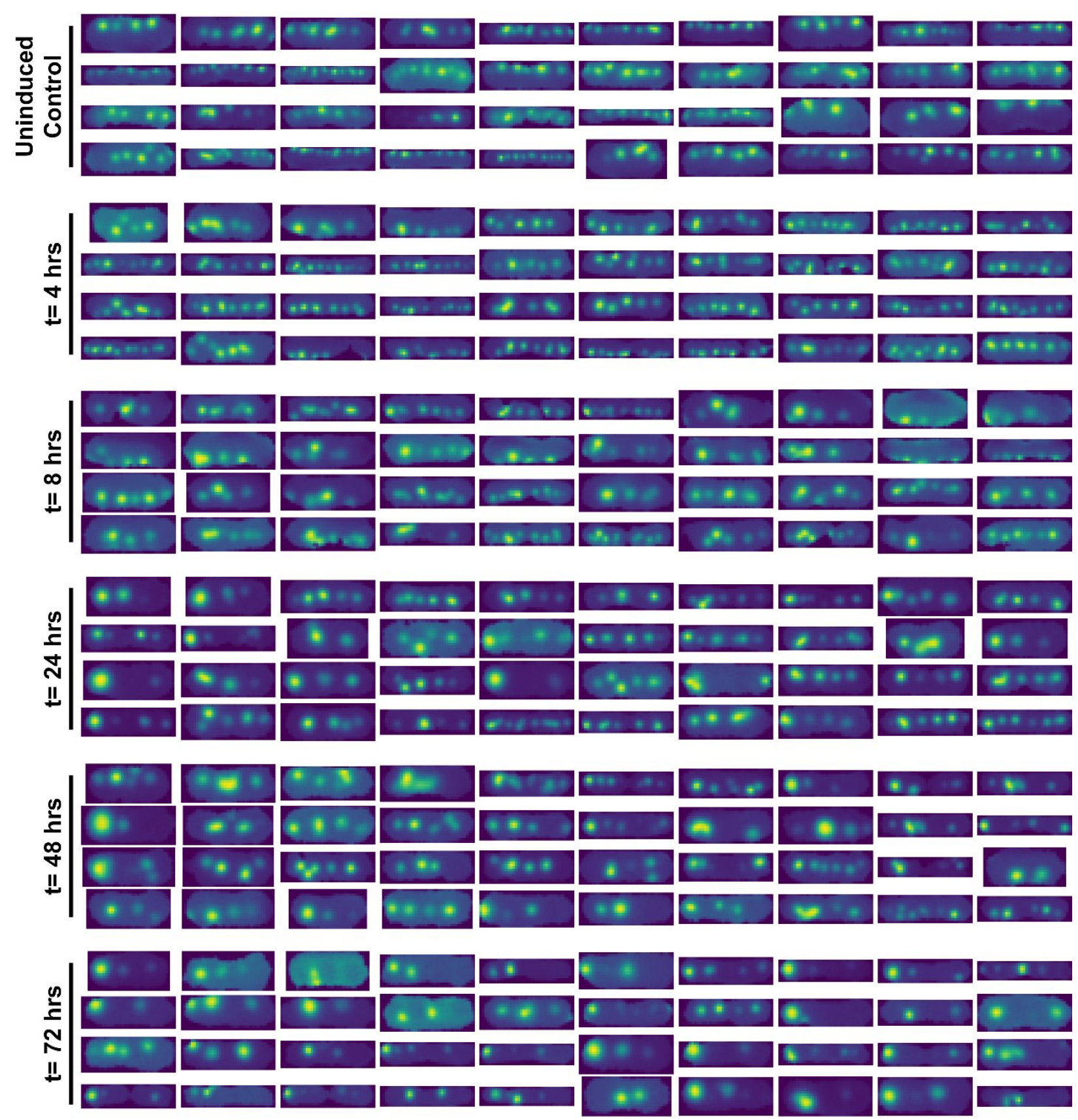

Figure S12: Representative segmented cells collected from a time series of CcmO-PDT downregulation. CcmO-PDT was targeted for downregulation by $m f$-lon protease in strain DD321 $(30 \mu \mathrm{M})$ and carboxysome morphology and positioning was followed up to 72 hours post induction. Cells were segmented using chlorophyll autofluorescence with a custom Python script, as described in the Materials \& Methods, and segmented cells were rotated so that the medial axis was horizontal to produce a random sample of cell images, representative of those used for quantitative measurements (e.g., inter-carboxysome spacing). Fluorescence from the RbcS reporter (mNG channel) shown using a pseudo-color 'heat map' (dark blue $=$ low fluorescence, yellow $=$ high fluorescence). While dramatic restructuring of carboxysomes is evident in later time points after CcmO-PDT downregulation, subtle phenotypes are qualitatively evident 
at earlier timepoints (e.g., 4 hours post-induction). This includes an increased heterogeneity in the size and brightness of carboxysomes within each cell. For reference, a sample from pooled uninduced control images (all time points) are included (top). Cells containing $\leq 1$ carboxysome (polar body phenotype) were excluded from these images, $n=20$ per time point. Strains: CcmO-PDT/mf-lon/RbcS-mNG (DD321).

A
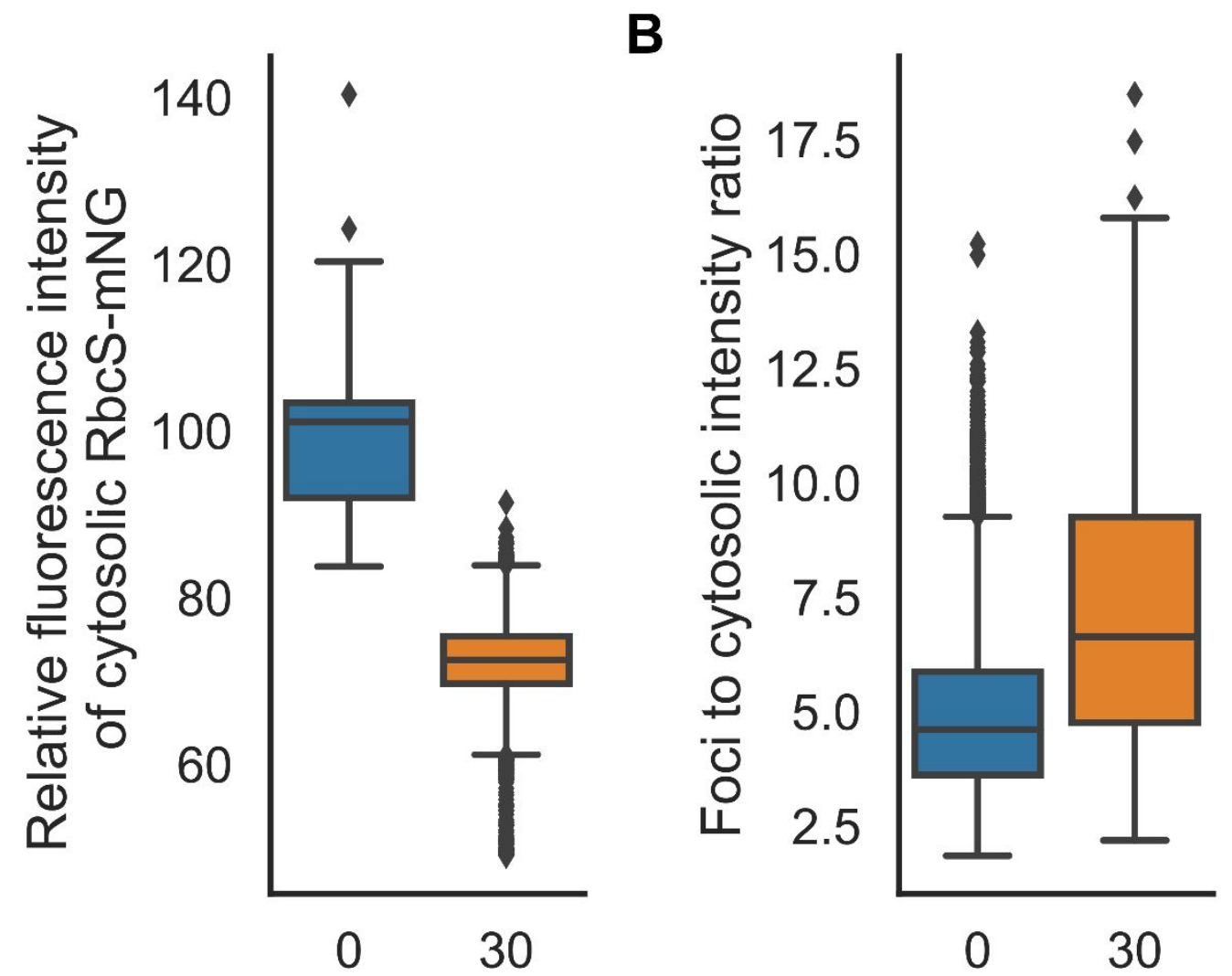

\section{Theophylline concentration $(\mu M)$}
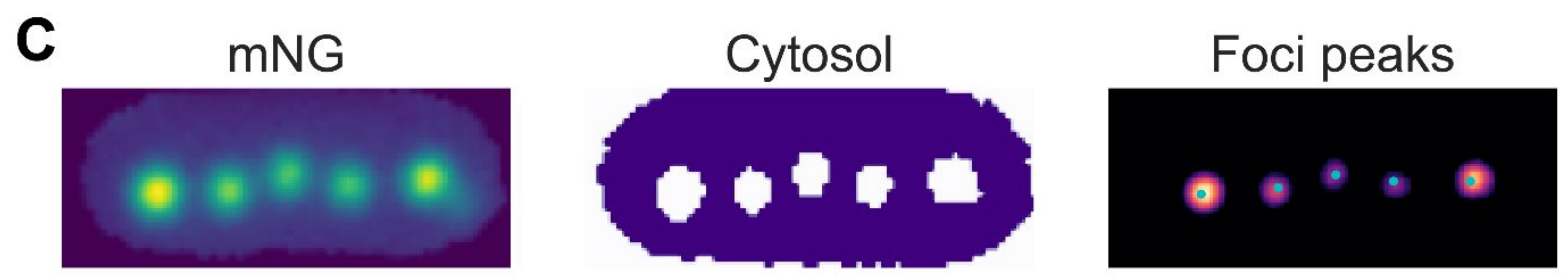

Figure S13: Cytosolic Rubisco reporter is depleted 72 hours following mf-lon-mediated carboxysome shell degradation. A) Relative cytosolic Rubisco reporter (RbcS-mNG) fluorescence in Strain DD321, normalized to uninduced control at $t=0$ hours. B) Ratio of peak foci to cytosolic intensity. Foci locations were determined with a peak-finding algorithm using the Python package Photutils ${ }^{3}$. C) Representative image of the RbcS- 
mNG fluorescence (left), area used in background cytosol analysis (middle), and foci with peak locations denoted in cyan (right). $n>1,700,2,300$ for panels $A$ and B, respectively. Strain: CcmO-PDT/mf-lon/RbcSmNG (DD321) 


\begin{tabular}{|l|c|c|c|}
\hline GO Term & P-Value & \# Hits & $\begin{array}{c}\text { \% Associated } \\
\text { Genes }\end{array}$ \\
\hline Ribosome & $2.33 \mathrm{E}-03$ & 6 & 10.53 \\
\hline Photosystem I & $2.62 \mathrm{E}-04$ & 4 & 30.77 \\
\hline Membrane protein complex & $3.65 \mathrm{E}-07$ & 12 & 14.46 \\
\hline Photosynthetic membrane & $1.87 \mathrm{E}-06$ & 12 & 12.12 \\
\hline Phycobilisome & $3.22 \mathrm{E}-09$ & 8 & 50.00 \\
\hline
\end{tabular}

Table S1: Gene ontology enrichment analysis of the off-target proteins downregulated following strong $\mathrm{mf}$ Ion protease over-expression in $S$. elongatus. Genes with statistically significant changes after a KruskalWallis Test $(p<0.05)$ and Benjamini-Hochberg correction $(p<0.00187), 41$ in total, were analyzed for enrichment. Phycobilisome-related genes (blue) were the most strongly enriched protein family of endogenous targets of $\mathrm{mf}$-lon protease. 


\section{Methods S1}

\section{Proteolytic Digestion \& TMT Labeling}

For the analysis of impacts on the proteome of overexpression of $m f$-lon protease, samples were induced with $1 \mathrm{mM}$ theophylline and cell samples were collected at time points 0,2 , and 4 hours after induction. 120 $\mu \mathrm{g}$ of protein sample for each time point (and for negative controls where no inducer was added) was precipitated using acetone. Protein pellets were re-suspended in $270 \mu \mathrm{L}$ of $100 \mathrm{mM}$ ammonium bicarbonate supplemented with $10 \%$ trifluoroethanol. Samples were reduced and alkylated by adding tris(2carboxyethyl)phosphine (TCEP) and lodoacetamide at $10 \mathrm{mM}$ and $40 \mathrm{mM}$, respectively and incubated for $5 \mathrm{~min}$ at $45 \mathrm{C}$ with shaking at $1400 \mathrm{rpm}$. Trypsin/LysC enzyme mixture (V5071, Promega) in 100mM ammonium bicarbonate, was added at a $1: 100$ ratio $(\mathrm{wt} / \mathrm{wt})$ and the mixture was incubated at $37^{\circ} \mathrm{C}$ overnight. Final volume of each digest was $\sim 300 \mu \mathrm{L}$. After digestion, the samples were acidified to $2 \%$ TFA and subjected to $\mathrm{C} 18$ solid phase clean up using StageTips ${ }^{4}$ to remove salts. Eluates were dried using a vacuum centrifuge. Peptide samples were then re-suspended in $100 \mu \mathrm{L}$ of $100 \mathrm{mM}$ tetraethylammonium bicarbonate (TEAB) and labeled with TMT reagents (Thermo Scientific) according to manufacturers' instructions. A $2 \mu \mathrm{L}$ aliquot of each labeled peptide sample was taken for label incorporation testing. The remainder of each labeled sample was combined in equal amounts by volume and purified by solid phase extraction using 18 SepPak cartridges (Waters). Eluted peptides were dried by vacuum centrifugation to $\sim 2 \mu \mathrm{L}$ and stored at $-20^{\circ} \mathrm{C}$. Purified peptides were re-suspended in $2 \%$ acetonitrile/ $0.1 \%$ trifluoroacetic acid (TFA) to $20 \mu \mathrm{L}$.

\section{LC/MS/MS Analysis}

An automatic $5 \mu \mathrm{L}$ injection was controlled via a EASYnLC 1200 onto an Acclaim PepMap RSLC $0.1 \mathrm{~mm} x$ $20 \mathrm{~mm}$ C18 trapping column and washed for $\sim 5 \mathrm{~min}$ with buffer $\mathrm{A}$ (ThermoFisher Scientific; Buffer $\mathrm{A}=99.9 \%$ water $/ 0.1 \%$ formic acid, Buffer $B=80 \%$ acetonitrile $/ 0.1 \%$ formic acid $/ 19.9 \%$ water). Bound peptides were then eluted onto a Thermo Acclaim PepMap RSLC $0.75 \mathrm{~mm}$ x 500mm C18 analytical column over $125 \mathrm{~min}$ with a gradient of $5 \% \mathrm{~B}$ to $8 \% \mathrm{~B}$ in $5 \mathrm{~min}, 8 \% \mathrm{~B}-40 \% \mathrm{~B}$ in $109 \mathrm{~min}$, ramping to $90 \% \mathrm{~B}$ in $1 \mathrm{~min}$ and held at $90 \% \mathrm{~B}$ for the duration of the run at a constant flow rate of $300 \mathrm{~nL} / \mathrm{min}$. Column temperature was maintained at $50^{\circ} \mathrm{C}$ using an integrated column heater (PRSO, v2, Sonation). Eluted peptides were sprayed into a QExactive HF-X mass spectrometer using a FlexSpray spray ion source (ThermoFisher Scientific). Survey scans were taken in the Orbi trap (120,000 resolution, determined at $\mathrm{m} / \mathrm{z} 200)$ and the top 15 ions in each survey scan are then subjected to automatic higher energy collision induced dissociation (HCD) with fragment spectra acquired at 45,000 resolution. The resulting MS/MS spectra were converted to peak lists using ProteomeDiscoverer, v2.2.0 (ThermoFisher Scientific) and searched against a protein database containing all S. elongatus entries found in UniProt (downloaded 2019-10-11; ${ }^{5}$ ) using the Mascot searching algorithm, v 2.6, within ProteomeDiscoverer. The Mascot output was then analyzed using Scaffold Q+S, v4.10.0 (S) to probabilistically validate protein identifications with the X!Tandem validation search option selected. Assignments validated using the Scaffold 1\%FDR confidence filter are considered true. 


\section{References:}

(1) Jordan, A.; Chandler, J.; MacCready, J. S.; Huang, J.; Osteryoung, K. W.; Ducat, D. C. Engineering Cyanobacterial Cell Morphology for Enhanced Recovery and Processing of Biomass. Applied and Environmental Microbiology 2017, 83 (9), 113. https://doi.org/10.1128/aem.00053-17.

(2) Cameron, J. C.; Wilson, S. C.; Bernstein, S. L.; Kerfeld, C. A. Biogenesis of a Bacterial Organelle: The Carboxysome Assembly Pathway. Cell 2013, 155 (5), 1131-1140. https://doi.org/10.1016/j.cell.2013.10.044.

(3) Bradley, L.; Sipőcz, B.; Robitaille, T.; Tollerud, E.; Vinícius, Z.; Deil, C.; Barbary, K.; Wilson, T. J.; Busko, I.; Donath, A.; Günther, H. M.; Cara, M.; Conseil, S.; Bostroem, A.; Droettboom, M.; Bray, E. M.; krachyon; Lim, P. L.; Bratholm, L. A.; Barentsen, G.; Craig, M.; Rathi, S.; Pascual, S.; Perren, G.; Georgiev, I. Y.; ValBorro, M. de; Kerzendorf, W.; Bach, Y. P.; Quint, B.; Souchereau, H. Astropy/Photutils: 1.1.0. 2021. https://doi.org/10.5281/ZENODO.4624996.

(4) Rappsilber, J.; Mann, M.; Ishihama, Y. Protocol for Micro-Purification, Enrichment, Pre-Fractionation and Storage of Peptides for Proteomics Using StageTips. Nature Protocols 2007, 2 (8), 1896-1906. https://doi.org/10.1038/nprot.2007.261.

(5) Bateman, A. UniProt: A Worldwide Hub of Protein Knowledge. Nucleic Acids Research 2019, 47 (D1), D506-D515. https://doi.org/10.1093/nar/gky1049. 